\title{
A low-dimensional model of binocular rivalry using winnerless competition
}

\author{
Peter Ashwin \\ Mathematics Research Institute, University of Exeter, Exeter EX4 4QF, UK \\ Aureliu Lavric \\ School of Psychology, University of Exeter, Exeter EX4 4QF, UK
}

\begin{abstract}
We discuss a novel minimal model for binocular rivalry (and more generally perceptual dominance) effects. The model has only three state variables, but nonetheless exhibits a wide range of input and noise-dependent switching. The model has two reciprocally inhibiting input variables that represent perceptual processes active during the recognition of one of two possible states and a third variable that represents the perceived output. Sensory inputs only affect the input variables.

We observe, for rivalry-inducing inputs, the appearance of winnerless competition in the perceptual system. This gives rise to behaviour that conforms to well-known principles describing binocular rivalry (Levelt's propositions, in particular proposition IV: monotonic response of residence time as a function of image contrast) down to very low levels of stimulus intensity.
\end{abstract}

Key words: Binocular rivalry, perception, heteroclinic cycle, winnerless competition.

\section{Introduction}

One of the perceptual phenomena that have intrigued researchers over the years is that of "binocular rivalry" - when the stimuli presented to the

Email addresses: P.Ashwin@ex.ac.uk (Peter Ashwin), A.Lavric@ex.ac.uk (Aureliu Lavric) 
two eyes are different, perception alternates between the two stimuli roughly every few seconds [7]. The stimulus contrast is known to affect the predominance (average proportion of time spent perceiving one stimulus) and the dominance length/residence time (average time interval perceiving that stimulus). An influential synopsis of the influence of stimulus contrast on the dynamics of rivalry was proposed by Levelt [19] in his four propositions:

(I) "increase of the stimulus strength in one eye will increase the predominance of the stimulus";

(II) "increase of the stimulus strength in one eye will not [affect] dominance length for the same eye";

(III) "increase of the stimulus strength in one eye will increase the alternation frequency"; and

(IV) "increase of the stimulus strengths in both eyes will increase the alternation frequency."

To account for the empirical data on the role of stimulus properties in binocular rivalry $[19,4,5]$, as well as on its neurophysiological correlates $[9,11,18,29]$, a number of models have been put forward, most of which have been based on a reciprocal inhibition architecture, whereby the parts of the system that code for the two competing percepts suppress each other $[3,10,14,15,16,20,30]$. Dominance switching is typically instantiated via slow negative feedback (e.g. population adaptation or synaptic depression variables). This negative feedback reduces inhibition exerted by the dominant side over the suppressed one. Some of the modelling efforts have been directed at the Levelt propositions outlined above. For example, Laing and Chow [15] developed a population rate model (derived from a network model of Hodgkin-Huxley type neurons) containing two populations of neurons coding the competing percepts with reciprocal inhibitory connections between the two populations. In the model, the activity of the populations is sustained by recurrent excitation and reduced by population adaptation variables; the model also incorporates synaptic depression variables, which modulate the strength of recurrent excitation and that of reciprocal (mutual) inhibition between the competing populations. Laing and Chow [15] reported the model to be consistent with all Levelt's propositions, along with other empirical phenomena. 
However, recently Shpiro et al. [26] showed that the Laing and Chow model and other models based on mutual inhibition do not always conform to Levelt's propositions. In particular, [26] set out to examine the models' behaviour in relation to proposition IV (see above). Four models were subjected to scrutiny: the Laing and Chow model [15], two modified versions of this model (one without population adaptation and one without synaptic depression), and the model by Wilson [30]. Although the latter is mathematically different from the Laing and Chow model, the two share some important qualitative features: mutual inhibition between the model components representing the competing stimuli and the presence of a slow population adaptation process that is essential for the switching of dominance. Shpiro et al. [26] examined dominance durations for different stimulus contrasts, while also varying the values of the inhibition strength parameter. In addition, they assessed the effects of noise on the contrast-dominance duration relationship, the role of recurrent excitation (by omitting its term in the modified Laing and Chow models) and the effects of having a separate inhibitory population (present in the Wilson model and absent in the Laing and Chow model and its modifications).

The principal result was that none of the four models showed a monotonic dependence between stimulus contrast and dominance durations. The most common behaviour across models and inhibition strengths can be summarised as follows. For high stimulus contrast values, dominance duration decreased with increasing stimulus contrast as posited by Levelt's proposition IV (we will henceforth adopt Shpiro et al.'s terminology and refer to this as decreased duration, or DD, behaviour). For intermediate stimulus contrast values, no switching occurred, with one population active and other inactive indefinitely ("Winner Takes All" behaviour). For low stimulus contrast values, dominance durations increased with increasing stimulus contrast (increased duration, or ID, behaviour [26]). The "Winner Takes All" mode for intermediate stimulus contrast values was not found when the crosspopulation inhibition parameter had low values or when random white noise was introduced. However, the non-monotonic dependence between stimulus contrast and dominance duration was still observed (ID behaviour for lower contrasts and DD behaviour for higher contrasts). The presence of recurrent excitation (in the Laing and Chow model [15]) or that of a separate inhibitory population (in the Wilson model [30]) did not seem to influence the (nonmonotonicity of the) relationship between stimulus contrast and dominance durations. 
Another important finding of Shpiro et al. [26] concerned asymmetric input to the two eyes, which is relevant for Levelt's propositions I-III (see above). For the range of stimulus contrasts for which models showed DD behaviour, Shpiro et al. found that increasing stimulus contrast for one eye reduced dominance durations for the other eye's percept, without affecting the dominance durations of the stimulus whose contrast was changed (consistent with propositions I-III). However, for the range of stimulus contrasts associated with ID behaviour, the effect of increasing stimulus contrast for one eye was inconsistent with propositions I-III, leading to an increase in dominance durations for the same eye's percept.

The non-monotonic contrast-dominance relationship in all four models led [26] to highlight the mismatch between models and Levelt's propositions, particularly proposition IV. Our main contribution here is to produce a similarly motivated minimal model that nevertheless does produce "Levelt IV"type (DD) behaviour all the way down to arbitrarily small stimulus contrasts. The model also shows behaviour compatible with propositions I-III in conditions of asymmetric stimulus contrast. This is achieved by means of "Winnerless Competition" between perceptual states.

\subsection{Winnerless competition models for cognitive processes}

Models of neural dynamics tend to be variational (i.e. they have an energy landscape that is explored by the dynamics). However, there is no a priori reason why they should be, other than this leading to an elegant understanding of their dynamics in terms of minimization on the landscape. In particular, there may be novel dynamical mechanisms for neural processes that do not fit into the energy landscape paradigm. A particular example of non-variational dynamics called "Winnerless Competition" (WLC) [22, $23,24]$ was introduced by Rabinovich, Huerta and co-workers to explain a variety of switching-type responses and sequence generation for low-level neural microcircuits. Such dynamical models have robust attractors that are composed of a network of unstable states of saddle type connected by their unstable manifolds. The individual saddles appear to attract for a certain time, but any small components in the unstable directions grow, leading to eventual "switching" between saddles. In terms of nonlinear dynamics, such attractors have been studied for some time as heteroclinic networks and there is an extensive literature on their robustness, their stability (attractiveness) $[12,13,24]$ and structure $[1,21]$. 
Although WLC has been primarily developed for low level modelling (with few exceptions, e.g. [24]), it may be very useful for the modelling of higher level cognitive processes [2]. This paper presents a case study of a model for binocular rivalry that is built on winnerless competition principles. The model's architecture shares some common characteristics with the models discussed above. It contains two components which represent neuronal populations each of which responds to one of the two stimuli. Based on singlecell neurophysiological data showing more dominance switching responses in binocular cells in areas V4 and MT [18], we are inclined to assume that these model components are not groups of monocular cells in the Lateral Geniculate Nucleus or the primary visual cortex, but populations of binocular cells in higher visual areas that code the perceptual features present in a given stimulus (which happens to be presented to one eye). However, they could equally represent competing groups of monocular neurons, which would be more consistent with recent neuroimaging evidence of low-level inter-ocular competition in binocular rivalry [9, 29]. As in the models discussed above $[15,30]$, in our model the neuronal populations corresponding to the two stimuli compete via reciprocal inhibition; like these models, ours also incorporates an adaptation process, which leads to saturation of activity in the two populations. However, unlike the above-mentioned models, our model also contains a third, "arbitration", component. It could represent either a neuronal population that synthesises the inputs from modules specialised for particular stimuli/configurations, or the difference in activity between such specialised modules. In either case, the dynamics of the "arbitration" component underlies the perceptual state experienced (and reported) by the subject.

\section{A simple winnerless competition model of binocular rivalry}

We consider a simple model in $\mathbf{R}^{3}$ of the perceptual processes involved in binocular rivalry, with connectivity as illustrated in Figure 1. In this model we consider three dynamical variables that model the underlying population dynamics:

- The variables $x$ (resp. $y$ ) represent an activity pattern associated with the stimulus presented to the left (resp. right) eye.

- The variable $p$ represents the activity in the "arbitration" component, which underlies the reported perceptual state. 


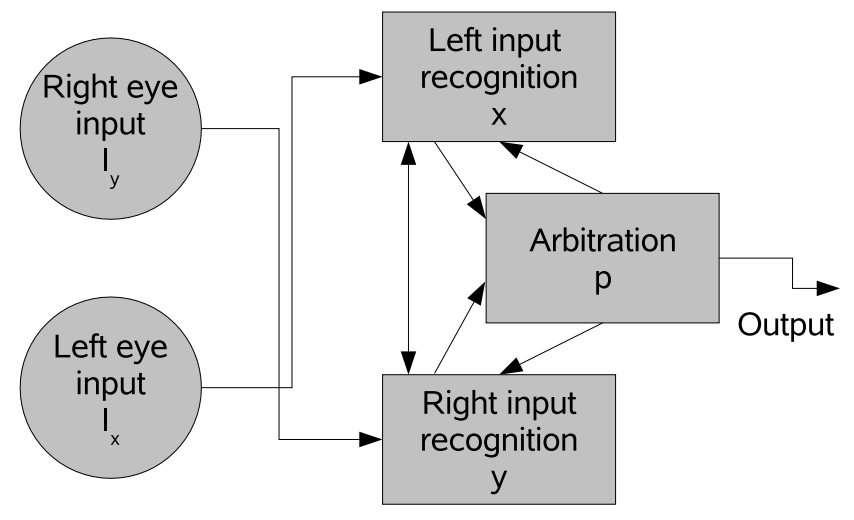

Figure 1: A simple network that can resolve binocular rivalry using winnerless competition.

We assume in the absence of $x$ or $y$ input that the dynamics of $p$ has two stable states, one where the left eye is dominant and one where the right eye is dominant. We assume that the dynamics of $x, y$ are usually at rest but, depending on input and currently perceived state, they can undergo a dynamical process (such as edge detection or comparison with a stored pattern) to attempt to recognize that particular pattern. If there is an input $I_{x}, I_{y}$ that indicates the presence of one of the "perceivable" states associated with $x, y$, then this results in growth of that variable.

In particular, if the input does not conform with the currently perceived pattern (represented by the current value of $p$ ) then the system dynamics will switch between the two possible states as shown schematically in Figure 2. The system we consider is:

$$
\begin{aligned}
& \dot{p}=h(p)+x^{2}(1-p)+y^{2}(-1-p)+\eta_{p} \\
& \dot{x}=f(p, x, y)+I_{x} x+\eta_{x} \\
& \dot{y}=g(p, x, y)+I_{y} y+\eta_{y}
\end{aligned}
$$

In (1) the quantities $I_{x}, I_{y}$ are inputs to the system for $x, y$ while the $\eta_{p}, \eta_{x}, \eta_{y}$ represent independent biased Wiener noise inputs. These consist of a constant bias $\mu_{p}, \mu_{x}, \mu_{y}$ added to an unbiased white noise with amplitude (i.e. variance growth per unit time) $\sigma_{p}, \sigma_{x}, \sigma_{y}$ respectively. We take

$$
h(p)=-p(p-1)(p+1)
$$




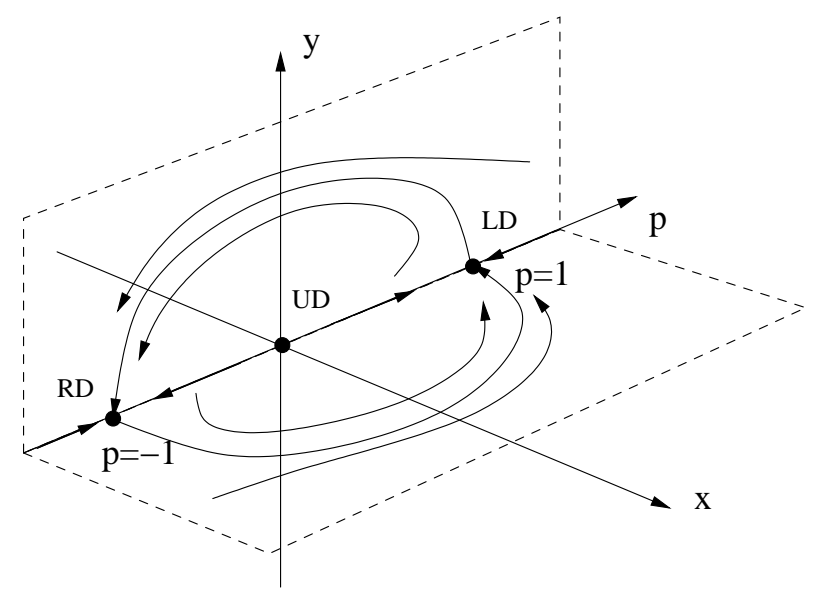

Figure 2: Schematic diagram showing the dynamics of the system for an input invoking binocular rivalry with $0<I_{x}, I_{y}$ and $\mu_{x}=\mu_{y}=\mu_{p}=0$. There are two saddle states on the axis $x=y=0$ that are only unstable to perturbations in one of the directions $x, y$ respectively. Typical initial conditions (not contained in one of the planes $x=0$ or $y=0$ ) approach the loop of unstable manifolds from $(p, x, y)=(-1,0,0)$ to $(p, x, y)=(1,0,0)$ and back.

to represent the dynamics of $p$ on the subspace $x=y=0$. One can write $h(p)=-V^{\prime}(p)$ where $V(p)=\frac{1}{4} p^{4}-\frac{1}{2} p^{2}$ represents a twin well potential with equal minima at $p= \pm 1$ as shown in Figure 3 .

The dynamics of $x, y$ are determined by the functions

$$
\begin{aligned}
& f(p, x, y)=\left[(0.5-p)(p+1)-x^{2}-y^{2}\right] x \\
& g(p, x, y)=f(-p, y, x) .
\end{aligned}
$$

The $p$-dependent terms in (2) are chosen such that for $I_{x}>0, p=-1$ has an instability to increasing $x$ while similarly for $I_{y}>0, p=1$ has an instability to increasing $y$. On the other hand, if $I_{x}<0$ (resp. $\left.I_{y}<0\right)$ this stabilizes $p=-1$ (resp. $p=1$ ) which means that the model (1) can represent a number of different modes of dynamical behaviour. The remaining terms in $f$ from (2) translate into a "generic" accommodation term $-x^{3}$ and a cross-inhibition term $-y^{2} x$.

\subsection{The dynamics of the model}

When the input recognition systems $x=y=0$ are not excited, the dynamics of $p$ is that of the twin-well potential flow, as illustrated in Figure 3. 

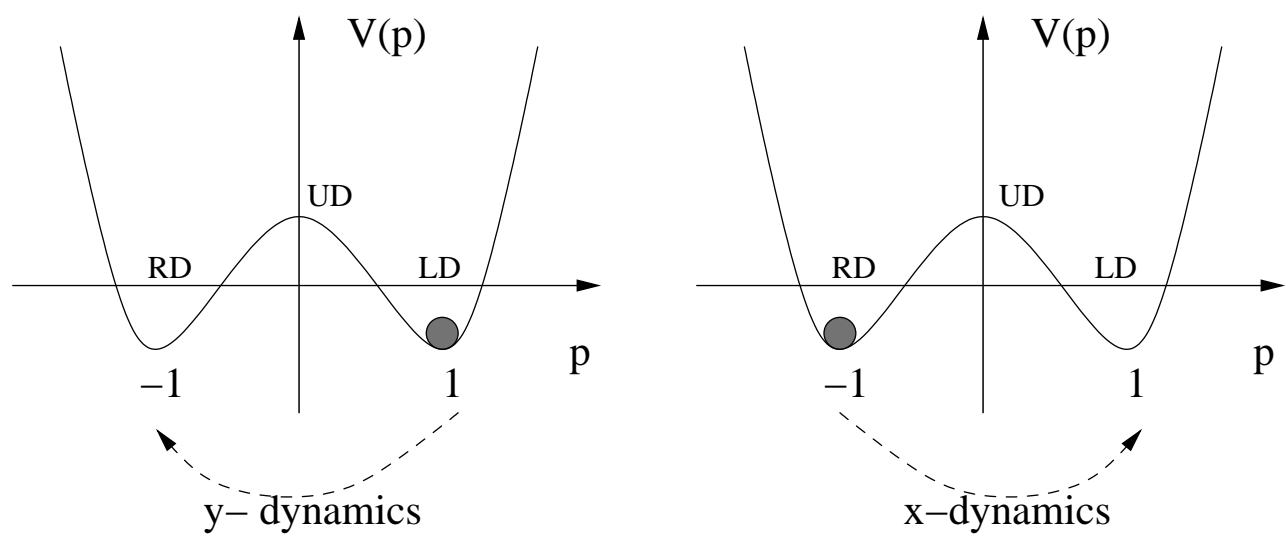

Figure 3: Schematic diagram showing the $p$ dynamics under a condition of binocular rivalry. In the case $x=y=0$ the dynamics of the perception variable $p$ as governed by a variational equation with potential $V(p)$ and remains at one of two possible bistable states. However, the two transverse variables $x$ and $y$ can induce a change of the state from one to the other. Note that the dynamics of the system is variational for $p$ on $x=y=0$ but is not variational away from this subspace.

In the absence of noise, and for $I_{x}=I_{y}, \mu_{x}=\mu_{y}$ and $\mu_{p}=0$ the model (1) has a symmetry

$$
(p, x, y) \mapsto(-p, y, x) .
$$

For $\eta_{x}=\eta_{y}=0$ the model has two invariant subspaces $x=0$ and $y=0$, but this is only approximate for other parameter values. For these idealised parameters there are three equilibria in $x=y=0$ : a left dominant $L D$ resting state $(p, x, y)=(1,0,0)$, a right dominant $R D$ resting state $(p, x, y)=$ $(-1,0,0)$ and an indeterminate unstable $U D$ resting state $(p, x, y)=(0,0,0)$. These states may or may not be stable to transverse $x, y$ dynamics and this can easily be determined by examining the linearized stability: in fact $L D$ becomes unstable to $y$ perturbations if $I_{y}>0$ while $R D$ becomes unstable to $x$ perturbations if $I_{x}>0$.

Figure 4 shows bifurcations of steady states in the model for symmetric inputs $I=I_{x}=I_{y}$ that is rivalrous for $I>0$. Observe that the $L D, R D$ states are bistable for $I<0$. For the range $0<I<0.5$ the only attracting dynamics of the model is that of winnerless competition; a robust heteroclinic cycle with alternation between $L D$ and $R D$ states. There is a transition to a stable indeterminate state $(0, x, x)$ for $I>0.5$; in fact there is still a robust heteroclinic cycle for all $I>0$ but this is no longer attracting for $I>0.5$. 


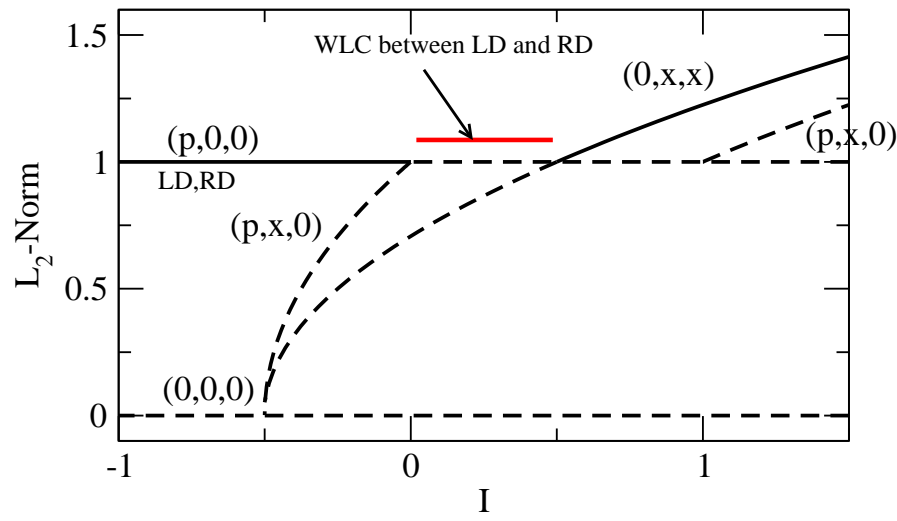

Figure 4: Bifurcation diagram showing steady branches of the noise-free system $(1,3)$ with input $I=I_{x}=I_{y}$, for $\mu_{p}=\mu_{x}=\mu_{y}=0$. Solid and dashed lines denote stable and unstable solutions, respectively. In the marked region $(0<I<0.5)$ there is robust winnerless competition dynamics: see Figure 5. In this region, addition of small but nonzero $\mu_{p} \mu_{x}$ and $\mu_{y}$ gives an attracting periodic orbit showing switching between $p=1$ and $p=-1$ states with period typically in Figure 6 .

The dynamical behaviour under WLC is shown in Figures 2 and 5. For an open set of initial conditions, trajectories move towards the unstable states $L D$ and $R D$ and their unstable manifolds; as time progresses they will switch between the two states, spending progressively longer at each state in turn. One can interpret the qualitative dynamics of (1) for WLC as follows: The $p$ dynamics in the absence of inputs $x, y$ is that of a twin-well potential with minima at $p=-1$ and $p=1$, and a separating saddle at $p=0$ (see Figure 3 ). Recognition by the left eye (activity of $x$ ) will push the $p$ dynamics towards $p=1$ while activity of $y$ will push it towards $p=-1$. In the case where $\mu_{x, y}$ non-zero and $\sigma_{p, x, y}=0$, this is an attracting periodic orbit whose period is plotted in Figure 6.

We choose "standard parameters" as follows

$$
\mu_{x}=\mu_{y}=10^{-4}, \quad \mu_{p}=0, \quad \sigma_{x}=\sigma_{y}=5 \times 10^{-5}, \quad \sigma_{p}=0.02,
$$

where we recall that $\eta_{p, x, y}$ denotes a biased Wiener noise with mean $\mu_{p, x, y}$ and amplitude (variance growth per unit time) $\sigma_{p, x, y}$. For such parameters (with non-zero $\mu_{x, y}$ ) we find that the WLC behaviour is replaced by an approximately periodic switching between $L D$ and $R D$ states, with mean switching period close to that for the noise-free case shown in Figure 6. 

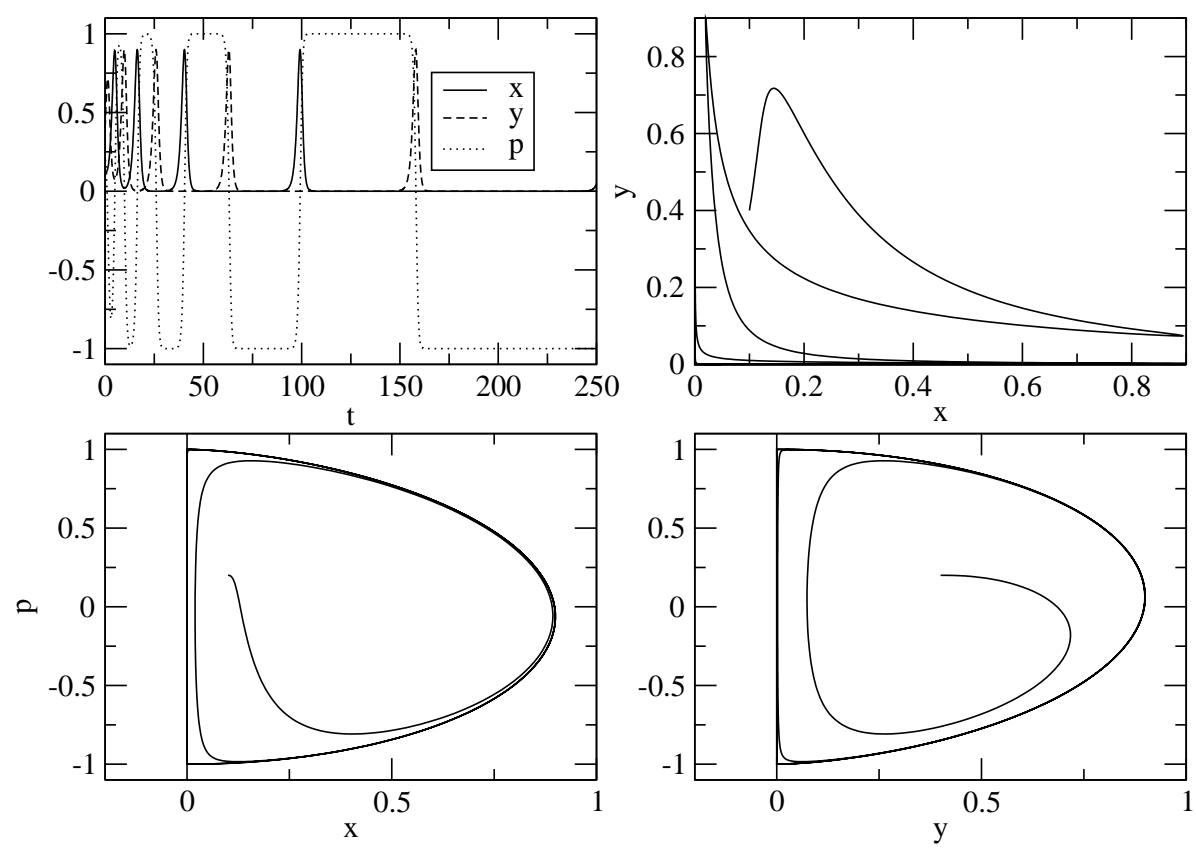

Figure 5: Dynamics of the system (1) in the absence of noise, for $\mu_{x}=\mu_{y}=\mu_{p}=0$ and $I_{x}=I_{y}=0.4$. There is an alternation between the two saddle states $L D(1,0,0)$ and $R D$ $(-1,0,0)$, with a progressive slowing down typical of an attracting heteroclinic cycle.

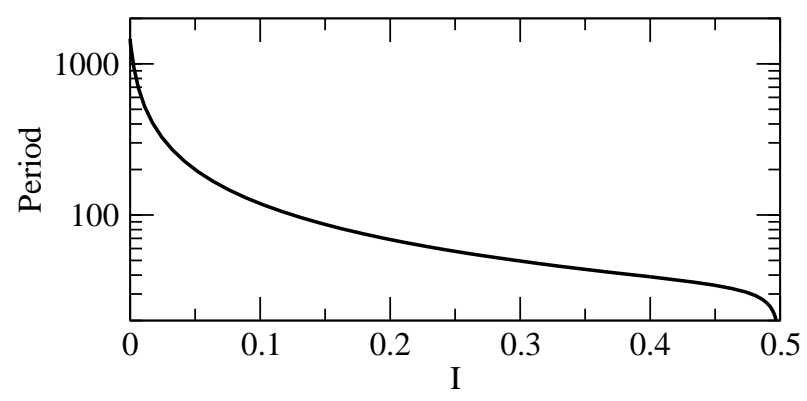

Figure 6: Period of the stable "alternating" WLC periodic orbit for the noise-free system (1) with rivalry-inducing input $I=I_{x}=I_{y}$, for $\mu_{x}=\mu_{y}=0.0001$ and $\mu_{p}=0$. Observe the decreasing dominance times (DD) for increasing input. 


\subsection{Scalings and quantitative properties of the system}

One can understand the behaviour near a robust heteroclinic cycle such as Figure 2 in the presence of symmetry breaking perturbations [25] or noise [28] by considering a singular perturbation of the heteroclinic cycle. Any noise/symmetry breaking will have most effect when the trajectory is close to one of the equilibria on the cycle, so we proceed by constructing a return map. For clarity, in this section we assume at first that both mean input and noise can be ignored.

Consider a small neighbourhood of $p=1$ and for some $\epsilon>0$ small, but significantly larger that the mean inputs, we take Poincare sections

$$
\begin{aligned}
H^{\text {in }+}=\left\{\left(1+q_{0}, \epsilon, y_{0}\right)\right\}, & H^{\text {out }+}=\left\{\left(1+q_{0}^{\prime}, x_{0}, \epsilon\right)\right\}, \\
H^{\text {in- }}=\left\{\left(-1+q_{1}, x_{1}, \epsilon\right)\right\}, & H^{\text {out }}=\left\{\left(-1+q_{1}^{\prime}, \epsilon, y_{1}\right)\right\}
\end{aligned}
$$

where $\epsilon$ is fixed and $q_{i}, x_{i}, y_{i}$ are variables that parametrize the Poincaré sections. We obtain an approximate return map $\phi_{+}: H^{\text {int }} \rightarrow H^{\text {out }+}$ from the linearized flow

$$
\left(\begin{array}{c}
\dot{q} \\
\dot{x} \\
\dot{y}
\end{array}\right)=\left(\begin{array}{ccc}
-2 & 0 & 0 \\
0 & -1+I_{x} & 0 \\
0 & 0 & I_{y}
\end{array}\right)\left(\begin{array}{l}
q \\
x \\
y
\end{array}\right)+\left(\begin{array}{l}
\mu_{q} \\
\mu_{x} \\
\mu_{y}
\end{array}\right) .
$$

The $q$ variable decouples from the linearization and does not affect the passage time, meaning we need to consider only the $x, y$ components starting at $\left(1+q_{0}, \epsilon, y_{0}\right) \in H^{i n+}$. We simplify the return map calculation by scaling to $\epsilon=1$, so that

$$
\begin{aligned}
& x(t)=\left(1-\frac{\mu_{x}}{1-I_{x}}\right) \exp \left(\left(-1+I_{x}\right) t\right)+\frac{\mu_{x}}{1-I_{x}}, \\
& y(t)=\left(y_{0}+\frac{\mu_{y}}{I_{y}}\right) \exp \left(I_{y} t\right)-\frac{\mu_{y}}{I_{y}} .
\end{aligned}
$$

Hence the first passage time from $H^{\text {int }}$ to $H^{\text {out+ }}$ is to leading order

$$
T_{x}=\frac{1}{I_{y}} \ln \left(\frac{1+\mu_{y} / I_{y}}{y_{0}+\mu_{y} / I_{y}}\right)
$$

and we exit at a point $\left(1+q_{0}^{\prime}, x_{0}, 1\right) \in H^{\text {out }+}$ where

$$
x_{0}=\left(1-\frac{\mu_{x}}{1-I_{x}}\right)\left(\frac{1+\mu_{y} / I_{y}}{y_{0}+\mu_{y} / I_{y}}\right)^{\left(-1+I_{x}\right) / I_{y}}+\frac{\mu_{x}}{1-I_{x}}
$$


if we assume $I_{x}, I_{y} \ll 1$ then to first order we have

$$
x_{0}=\left(1-\mu_{x}\right)\left(\frac{1+\mu_{y} / I_{y}}{y_{0}+\mu_{y} / I_{y}}\right)^{-1 / I_{y}}+\mu_{x} .
$$

Similarly the map from $H^{i n-}$ to $H^{\text {out- }}$ is characterised to lowest order by

$$
y_{1}=\left(1-\mu_{y}\right)\left(\frac{1+\mu_{x} / I_{x}}{x_{1}+\mu_{x} / I_{x}}\right)^{-1 / I_{x}}+\mu_{y} .
$$

We approximate the global maps from $H^{\text {out }}$ to $H^{\text {in- }}$ and from $H^{\text {out }}$ to $H^{i n+}$ by linear scalings $y_{0}=K y_{1}$ and $x_{1}=K x_{0}$ to first order.

If there is no noise present $\left(\sigma_{x}=\sigma_{y}=\sigma_{p}=0\right)$ and the quantities $x_{i}, y_{i}, I_{x}, I_{y}, \mu_{x}, \mu_{y}$ are small, then the transition time $T_{x}\left(\operatorname{resp} T_{y}\right)$ past the $p=1$ ( $\operatorname{resp} p=-1$ ) state can be estimated: we have $x_{0} \sim e_{x}=K \mu_{x}$ and $y_{1} \sim e_{y}=K \mu_{y}$ so that

$$
\begin{aligned}
& T_{x}=\frac{1}{I_{y}} \ln \left(\frac{1+\mu_{y} / I_{y}}{e_{y}+\mu_{y} / I_{y}}\right) \\
& T_{y}=\frac{1}{I_{x}} \ln \left(\frac{1+\mu_{x} / I_{x}}{e_{x}+\mu_{x} / I_{x}}\right) .
\end{aligned}
$$

The expression for $T_{x}$ only depends on input $I_{y}$, and shows DD behaviour over a wide range of inputs down to $I_{y}=0$. Closer examination indicates that for $I_{y}$ larger that $\mu_{y}$ the scaling is $T_{x} \sim 1 / I_{y}$, while as $I_{y} \rightarrow 0, T_{x}$ tends to a large (but finite) limit.

\section{Levelt's propositions I-IV for the model}

Numerical simulations of the model show that it conforms with Levelt's propositions I-IV over a wide range of rivalrous inputs $I_{x}, I_{y}>0$. Timestepping simulations of the model were undertaken on MATLAB using a fixed timestep 4th order Runge-Kutta integrator followed by an Euler stochastic step. The statistics of individual cases and statistics where checked using XPPAUT [8]. Figure 7 shows time series obtained from the model with standard parameters $(1,3)$ on changing inputs $I_{x}$ and $I_{y}$. A transient of 400 timesteps is excluded. In the figure we vary $I_{x} \in(0.1,0.2,0.4)$ (columns left to right) and $I_{y} \in(0.1,0.2,0.4)$ (rows top to bottom). In all cases one finds 
an approximately periodic switching between $p=-1$ and $p=1$, where the variation is mostly caused by the presence of noise in the system.

Figure 8 shows the durations of residence in the $p=-1$ and $p=1$ times for a typical case $\left(I_{x}, I_{y}\right)=(0.1,0.2)$. Observe the presence of two distributions with unequal mean. The variation of the mean residence times in the $L D, R D$ states are shown as bar graphs in Figure 9 for the parameter values used for Figure 7. For a higher level of noise input, Figure 10 shows in a longer run in with $I_{x}=I_{y}=0.1$ in $(\mathrm{a}, \mathrm{b})$; details in caption. Note that the distribution (c) of residence times in (b) spreads broadly around the mean value, and there is no evidence of correlation between residence times as illustrated by the rapid decay of autocorrelation $R(k)$ in (d). The distribution profile in (c) is shown along with best fits to both $\Gamma$ and $\log$ normal distributions ${ }^{1}$.

From Figure 9 we observe the conformity of the model to Levelt I-IV for this range of parameter values. Examining the graph for $p=-1$ ( $L D$ state) mean residence times clearly shows a strong dependence on the left input such that reducing the contralateral (right) input intensity $I_{x}$ increases the mean time spent in the $L D$ state, where there is only very weak dependence on ipsilateral (left) input intensity $I_{y}$. This conforms with propositions IIII. Finally, the diagonal also increases as $I_{x}=I_{y}$ is reduced indicating conformance to proposition IV.

Levelt's propositions I-IV can also be found from the approximate asymptotic formulae (6). Firstly, note that the asymptotic formulae are such that the dependence of the residence time is purely on the contralateral input (Levelt proposition II). Secondly, for fixed $e_{x}, e_{y}, \mu_{x}, \mu_{y}$ note that $T_{x}$ is a decreasing function of $I_{y}$ (Levelt proposition III) and one can identify two regimes. Clearly the same holds for $T_{y}$ dependence on $I_{x}$ and $\mu_{x}$.

\section{Conclusions}

The model seems to be one of the simplest ones where Levelt's propositions I-IV can be easily and controllably observed. As a low dimensional model it does not contradict the known mutual-inhibition architecture thought

\footnotetext{
${ }^{1}$ Best fit to a $\Gamma$ distribution $y=\left(x / a_{0}\right)^{a_{1}} \exp \left(-a_{2} x\right)$ has $a_{0}=17.48, a_{1}=$ $29.88, a_{2}=0.5298$ with Theil U-coeff 0.120547 best fit to log-normal distribution $y=$ $\left(a_{0} / x\right) \exp \left(-\left(\left(\ln (x)-a_{1}\right) / a_{2}\right)^{2}\right)$ has $a_{0}=9660, a_{1}=57.74, a_{2}=0.2477$ with Theil U-coeff 0.102357. Best fit to normal distribution has Theil U-coeff 0.1545.
} 

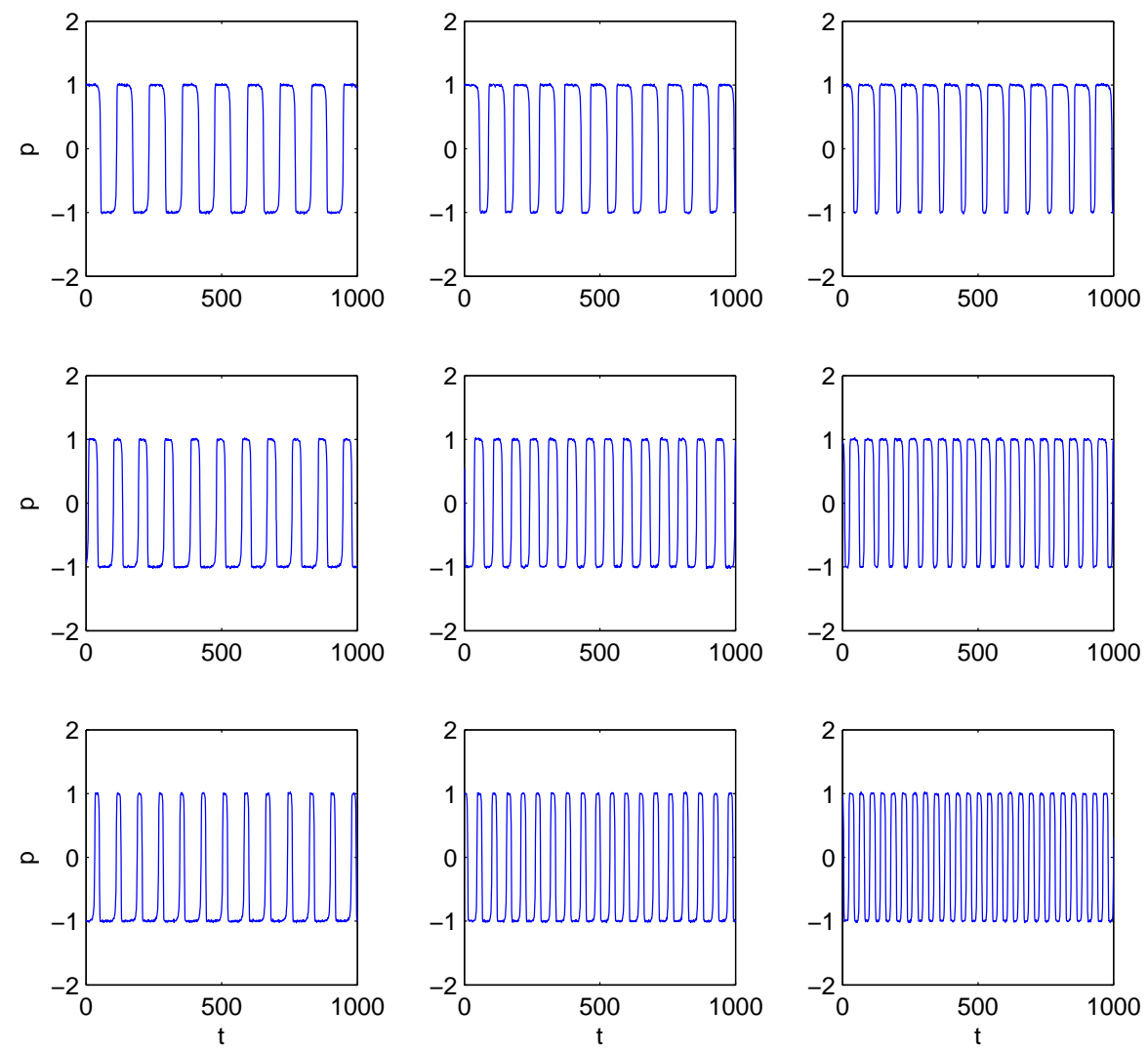

Figure 7: Time series showing $t$ evolution of $p$ for a range of $I_{x}$ and $I_{y}$ for the model $(1,3)$ after transients have decayed. The perceived output $p$ alternates between -1 and 1 while the other transverse variable are only non-zero during a transition. Columns: $I_{x}=0.1,0.2,0.4$ from left, Rows: $I_{y}=0.1,0.2,0.4$ from top. Mean residence times near $p=1$ and $p=-1$ are shown in Figure 9 . 




Figure 8: Distribution of residence times in the $p=-1$ and $p=1$ states for an extended run with $\left(I_{x}, I_{y}\right)=(0.1,0.2)$. Note that the mean residence time is clearly smaller for the $x$-preferred state $p=1$. Other details are as in Figure 7 .

to be responsible for rivalry effects. In particular, it is a model where propositions I-IV can exist over a large range of rivalry-inducing $0<I_{x}, I_{y}$.

The model predicts stable equilibrium dynamics for non-rivalrous inputs $I_{x}>0, I_{y}<0(p=1$ stable $)$ and $I_{x}<0, I_{y}>0(p=-1$ stable $)$ and for inputs that correspond to "Winner Takes All" bistable states $I_{x}<0, I_{y}<0$; see Figure 4. It would be interesting to extend the model in a number of ways, such as describing conditions under which the model might depart from Levelt's propositions I-IV (e.g. ID/DD phenomena) [26] and investigating additional phenomena such as dependence of "false return times" and "transition times" of Brascamp et al. [6] on inputs.

\subsection{Interpretation and relation to neurophysiologically-based models}

Unlike other models in the literature [26, 15, 30], we do not claim that our model is directly based on a neural substrates; rather it is a minimal low-dimensional model that explains several of the effects of binocular rivalry in terms of the dynamical paradigm of WLC. However, we note that the behaviour of $x, y$ has nonlinear saturation (i.e. adaptation) of their activity via $x^{3}, y^{3}$ and reciprocal inhibition via $x y^{2}, y x^{2}$ and so one can interpret the variables in a neurally plausible manner by considering the variables in (1) as measures of population activity. One intriguing correspondence between the dynamics of $x, y$ and empirical data is that either of them reaches maximum whilst in transition to, rather than during, the dominance interval of the respective percept (e.g. $x$ is maximal immediately before dominance switches to the left eye), see Figure 5. In electrophysiological recordings, 


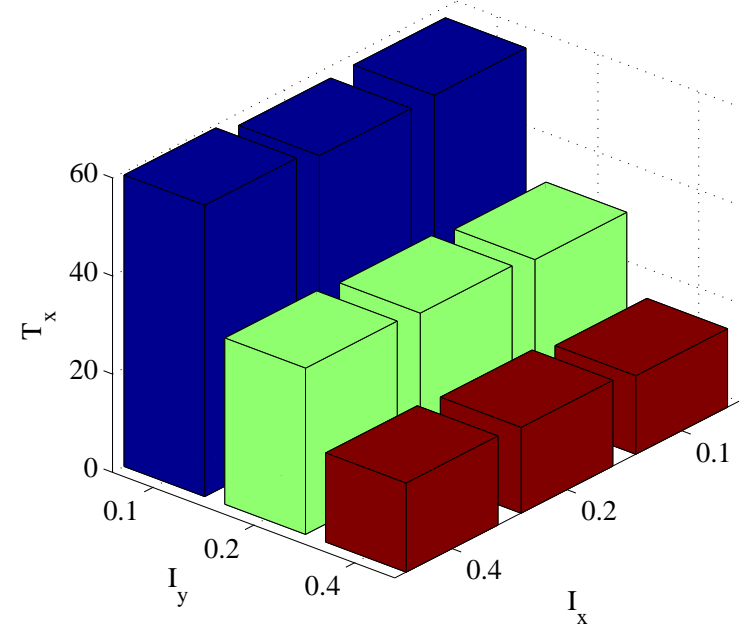

(a)

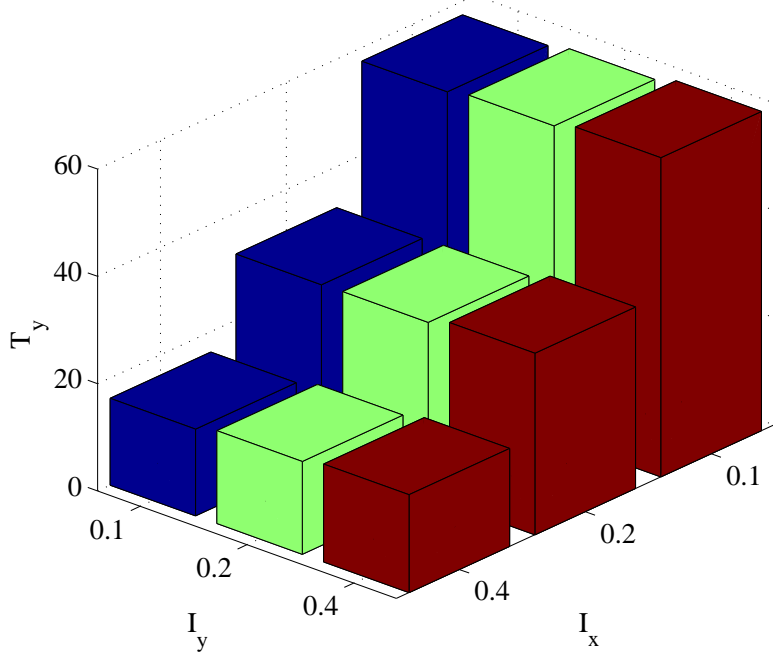

(b)

Figure 9: Average residence times (a) $T_{x}$ for $L D$ left dominated $(p=1)$ and (b) $T_{y}$ for $R D$ right dominated $(p=-1)$ state, over a range of inputs $I_{x}, I_{y}$. Other details are as in Figure 7. 

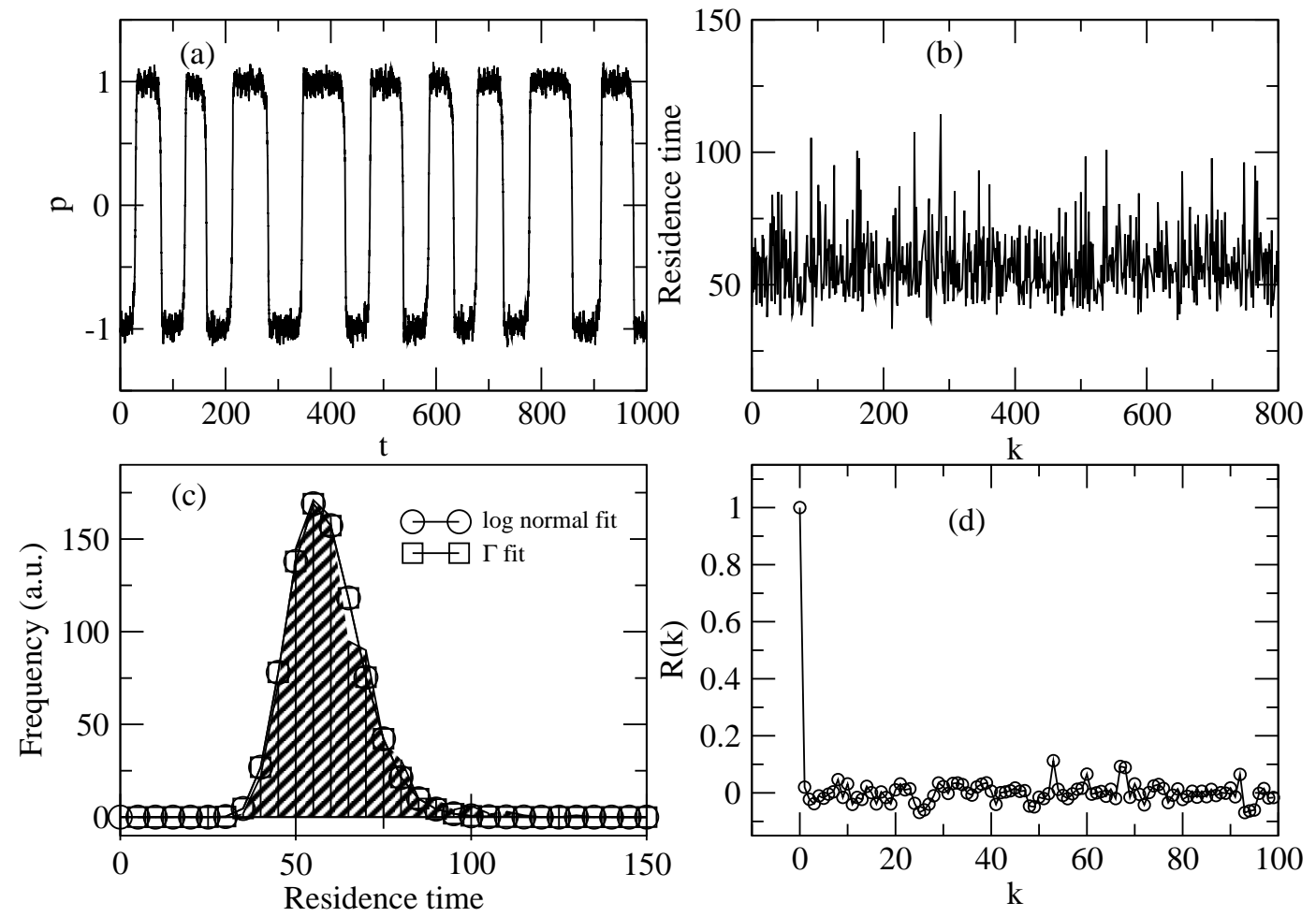

Figure 10: Dynamics of switching between $p=-1$ and $p=1$ states for a long run with symmetric input $I_{x}=I_{y}=0.1, \mu_{x}=\mu_{y}=\mu_{p}=0$ and higher noise; $\sigma_{x}=\sigma_{y}=0.001$ and $\sigma_{p}=0.1$. (a) shows part of a timeseries of switching, (b) gives a sequence of successive residence times in either state, (c) shows a frequency histogram $(N=800)$ of the data in (b) while (d) plots the dependence of the autocorrelation of the data in (b) against shift $k$. Observe the spread of residence times about the mean in (c) with good fits to both $\Gamma$ and log-normal distributions, and the independence of residence times exhibited by immediate decay of correlation to the noise floor, consistent with empirical data [17], in (d). 
rivalry-sensitive cells also show maximal spike rates right before the dominance switches to the cell's preferred stimulus. [18].

\subsection{Other applications for WLC models}

There are other cognitive dynamical effects (perceptual rivalry) that generalise the idea of binocular rivalry; these could similarly be modelled using the system (1) for suitable parameters. As noted in [24] WLC models and closely related "stable heteroclinic sequence" (SHS) dynamics may be a useful paradigm for understanding many forms of cognitive dynamics, including higher-order cognitive processes such as decision making. It has also been suggested [24] that SHS may be responsible for fluctuations seen in cortical resting states.

The model presented here suggests a model for rivalry with more than two percepts, generalising from bi-stability to multi-stability. One can make the $p$ dynamics more rich, including an attractor for each percept and introduce an extra "transverse" variable associated with each attractor. This transverse variable can be used to introduce transitions between percepts in the presence of rivalrous inputs. Figure 11 illustrates this idea schematically; by analogy with (1) the transverse variable depends on sensory inputs while

the "perception space" depends only on the transverse variables and initial condition.

\section{Acknowledgement}

PA thanks the EPSRC Mathematical Neuroscience Network, Rodica Curtu, Özkan Karabacak and Carlo Laing for their helpful advice.

\section{References}

[1] P. Ashwin and M. Field, Heteroclinic networks in coupled cell systems, Arch. Rational Mech. Anal. 148: 107-143, 1999.

[2] P. Ashwin and M. Timme, When instability makes sense, Nature, 436: 36, 2005.

[3] R. Blake, A neural theory of binocular rivalry, Psychol. Rev. 96: 145167, 1989.

[4] R. Blake and R. Fox, Adaptation to invisible gratings and the site of binocular rivalry suppression, Nature 249: 488-490, 1974. 




Figure 11: A generalization of the model discussed for binocular rivalry to a system with multiple perceptual states that live in a "perception space". These are governed by variational dynamics that drives the state into one of a number of possible percepts labelled $a, b, c, \ldots$. Mutually inhibitory "transverse" dynamics uses sensory inputs to temporarily stabilise one out of a number of possible percepts; in this diagram dynamics in $A$ drives the system via a heteroclinic connection to $a, B$ to $b$ etc.

[5] R. Blake, K. Yu, M. Lokey and H. Norman, Binocular rivalry and visual motion, J Cogn Neurosci. 10: 46-60, 1998.

[6] J.W. Brascamp, R. van Ee, A.J. Noest, R.H.A.H. Jacobs, A.V. van den Berg, The time course of binocular rivalry reveals the fundamental role of noise. Journal of Vision 6: 1244-1256, 2006.

[7] B.B. Breese, Binocular rivalry, Psychol. Rev. 16:410-415, 1909.

[8] G.B. Ermentrout. A Guide to XPPAUT for Researchers and Students. SIAM, Pittsburgh, 2002.

[9] J.D. Haynes, R. Deichmann, G. Rees, Eye-specific effects of binocular rivalry in the human lateral geniculate nucleus. Nature 438: 496-499, 2005.

[10] G.J. Kalarickal and J.A. Marshall, Neural model of temporal and stochastic properties of binocular rivalry, Neurocomputing 32-33: 843-853, 2000. 
[11] G. Kreiman, I. Fried, C. Koch, Single-neuron correlates of subjective vision in the human medial temporal lobe, Proc. Natl Acad. Sci. USA 99: 8378-8383, 2002.

[12] M. Krupa, Robust heteroclinic cycles, Journal of Nonlinear Science 7: 129-176, 1997.

[13] M. Krupa and I. Melbourne. Asymptotic stability of heteroclinic cycles in systems with symmetry, II. Proc. Roy. Soc. Edinburgh A 134A: 1177-1197, 2004.

[14] L. F. Lago-Fernández and G. Deco. A model of binocular rivalry based on competition in IT. Neurocomputing 44-46C: 503-507, 2002.

[15] C.R. Laing and C.C. Chow, A spiking neuron model for binocular rivalry. J Comput Neurosci. 12: 39-53, 2002.

[16] S.R. Lehky, An astable multivibrator model of binocular rivalry, Perception 17: 215-228, 1988.

[17] S.R. Lehky, Binocular rivalry is not chaotic, Proceedings of the Royal Society of London Series A 259: 71-76, 1995.

[18] D.A. Leopold and N.K. Logothetis, Activity changes in early visual cortex reflect monkey's percepts during binocular rivalry. Nature $\mathbf{3 7 9}$ : 549-553, 1996.

[19] W.J.M. Levelt, On binocular rivalry; In: Minor Series 2. Psychological Studies. The Hague: Mouton, 1968.

[20] E.D. Lumer, A neural model of binocular integration and rivalry based on the coordination of action-potential timing in primary visual cortex. Cerebral Cortex 8: 553-561, 1998.

[21] C.M. Postlethwaite and J.H.P. Dawes, A codimension-two resonant bifurcation from a heteroclinic cycle with complex eigenvalues. $D y$ namical Systems, 21: 313-336, 2006.

[22] M.I. Rabinovich, R. Huerta, P. Varona and V.S. Afraimovich, Generation and reshaping of sequences in neural systems. Biol. Cybern. 95: 519-536, 2006. 
[23] M.I. Rabinovich, R. Huerta and G.Laurent, Transient Dynamics for Neural Processing Science, 321: 48-50, 2008.

[24] M.I. Rabinovich, R. Huerta, P. Varona, V.S. Afraimovich, Transient Cognitive Dynamics, Metastability, and Decision Making, PLoS Computational Biol. 4: e1000072, 2008.

[25] B Sandstede and A. Scheel, Forced symmetry breaking of homoclinic cycles, Nonlinearity, 8: 333-365, 1995.

[26] A. Shpiro, R. Curtu, J. Rinzel, and N. Rubin, Dynamical characteristics common to neuronal competition models, J Neurophysiol. 97: 462-473, 2007.

[27] L. Stollenwerk and M. Bode, Lateral neural model of binocular rivalry. Neural Computation 15: 2863-2882, 2003.

[28] E. Stone and P. Holmes, Random perturbations of heteroclinic attractors, SIAM J. Appl. Math. 50: 726-743, 1991.

[29] F. Tong, S.A. Engel, Interocular rivalry revealed in the human cortical blind-spot representation, Nature 411: 195-199, 2001.

[30] H.R. Wilson, Computational evidence for a rivalry hierarchy in vision. Proc. Natl Acad. Sci. USA, 100: 14499-14503, 2003. 